\title{
Investigating implantable glucose biosensors pitfalls: a fault tree analysis approach
}

\author{
C. G. Siontorou \& F. A. Batzias \\ Department of Industrial Management and Technology, \\ University of Piraeus, Greece
}

\begin{abstract}
Implantable sensors for glucose monitoring are the first step towards the development of an implantable closed-loop diabetes control system. Although significant advances in the designs and chemistries employed to prepare intravascular and subcutaneous devices have been achieved, the biological responses can have a dramatic impact on the analytical accuracy of such probes. With a view to assisting the effective design of such devices for assuring clinical performance, the causes of implantable glucose sensor failure have been investigated by means of Fault Tree Analysis (FTA) relying on fuzzy reasoning to account for uncertainty. The approach suggested may contribute significantly to the self-optimisation of the measuring equipment from one generation to the next as it supports the flexible, ad hoc, and tailor made sensor development, thus potentiating the progress of epidemics from statistics to individualisation.

Keywords: biosensors, fault detection, fuzzy FTA, knowledge processing, sensitivity decrease, subcutaneous monitoring.
\end{abstract}

\section{Introduction}

Intensive treatment and close diabetes mellitus monitoring can prevent progression of long-term disease complications, such as retinopathy, nephropathy, and neuropathy. Accurate and systematic glucose monitoring is a key element for allowing patients to keep their glucose levels under control and for providing physicians with a more correct and detailed picture of the disease. The available self-monitoring devices, based on discrete monitoring measurements, apart from causing patients' discomfort, do not allow an overall, clear and in-depth view of the actual glycaemic status, especially in type I 
diabetics, where glycaemia may undergo rapid changes [1]. A continuous and fully automated subcutaneous glucose monitoring device would most certainly improve diabetic control, especially in less-apt individuals (young, elderly, mentally-challenged, etc.). It could also provide valuable information to R\&D regarding in vivo kinetic parameters after lunch, during sleep or exercise, as a first step towards a completely closed-loop control system that automatically monitors and maintains euglycaemia (artificial pancreas).

Numerous implantable miniaturized sensors have been developed, mostly on an electroenzymatic approach: using the oxidation of glucose by glucose oxidase, the produced hydrogen peroxide is measured either amperometrically or potentiometrically. Most sensors use a platinum cathode and a silver anode to form part of an electric circuit in which hydrogen peroxide is electrolyzed; the current through the circuit provides a measure of the hydrogen peroxide concentration, and hence of the glucose concentration in the vicinity of the sensor. For subcutaneous implantation, the sensors are designed with standard integrated circuit (IC) micro-fabrication processes; all signal processing can be accomplished on-board the chip, and the entire package is not more than a few square millimeters in size. Subcutaneous implantation of the sensors could be accomplished as an outpatient procedure with minor discomfort to the patient.

Unfortunately, the response of these devices undergoes a significant decrease (accompanied by a corresponding noise increase) when exposed to the biological environment, attributed to (i) biocompatibility issues: inflammation, fibrosis, scar formation, clot formation in blood vessels, or other tissue reactions $[2,3]$, (ii) biofouling: excessive protein deposition (clogging of the membrane pores with proteins resulting in a decrease or cessation of the flow of molecules to the sensor) [4-6], (iii) corrosion: decreased chemical resistance (the tissue environment can be remarkably corrosive) [7, 8], (iv) the inherent sensor pitfalls: problems associate with the biorecognition/transducer interface [7, 8], and (v) biocorrelation issues: the in vivo sensor performance differs significantly from its operation ex vivo, usually expressed as a rapid and significant sensor drift [9].

In view of (a) the inter-disciplinary and effectively diverse nature of the subject, (b) the complexity presented by the specific application and (c) the severity and prospects of the application, these devices require inevitably an intelligent system for real-time fault detection and fault compensation, an area which has not received yet much attention. The real-time response of such a system is imperative when considering that misleading measurements may enable inappropriate drug delivery that may stimulate vicious feed-back cycles, threatening to the life of the patient (e.g. severe hypoglycaemic episodes).

The identification of the possible sensor fault modes and their symptoms is a prerequisite for constructing such a system, requiring in-depth knowledge of sensor's design and operation, both from the electrochemical and the physiological/biochemical point of view. The most usual and critical fault mode in subcutaneous biosensors is sensitivity drift, which results either to an inaccurate measurement or to a dead sensor [7]. The environment within which the sensor operates, involving complex multi-component chemical interactions 
within multi-phase microstructures, increases uncertainty manifold since there could be (and usually are) many influences acting simultaneously or consecutively, masking, partially or totally, the identification of the problem source. There have been several attempts for understanding the possible failure mechanisms responsible for the poor in vivo performance of subcutaneously implanted glucose sensors, but still there is no clinical concept available and more insight is required in the physiological processes at the sensor-tissue interface. Limited information and high uncertainty has been successfully handled in several biomedical cases with fuzzy logic [10, 11]. Fuzzy set theory provides a way to use imprecise and uncertain information generated by the environmental system and the human judgment in a satisfactory way, in cases where absolute criteria should be avoided and the intuitive aspects of the experts' judgment should be preserved.

The work presented herein investigates the causes of implanted biosensor sensitivity reduction by means of Fault Tree Analysis (FTA), using the information provided by the biosensor components, the surrounding tissue and their intra/inter-relations on both, the surface- and the deep-knowledge level, simulating an expert system, where the tree (dendritic) structure (with the corresponding to each node appropriate confirmation tests and remedial proposals/advices) serves as the Knowledge Base (KB) and the fuzzy rules based decision mechanism on aetiology is the inference engine.

\section{Methodology}

The methodology adopted relies mainly on the design and the development of a hierarchical structure capable to perform Fault Tree Analysis (FTA) within a framework incorporating (a) the trunk of the SNR-decrease fault tree (i.e., the content part of the fault analysis), and (b) a mechanism of (i) assigning fuzzy partition of the space of the variables represented by each tree node and (ii) setting/revising of fuzzy rules used as an inference engine (i.e., the formal part of fault analysis).

The tree is structured in a top-down direction by deduction, involving the identification of the events or series of events that directly contribute to sensor malfunction, the immediate causes of these events and so forth, until the ultimate or final causes are identified. Conversely, a revision can be made by induction, after proper information has been collected. This dialectic trade-off between deduction and induction brings the tree design closer to scientific logic, permitting the introduction of deeper knowledge into the surface or experiential knowledge level characterizing FTA.

Once the final form (in content and architecture) of the fault tree has been agreed upon, the partitioning of the space of variables and the determination of the fuzzy rules serving as an inference engine for diagnosis can been achieved by using experts' opinion through a modified 4-stage Delphi method [7, 8]. Running the fuzzy FTA bottom-up, using first the combination of the final events that contributes mostly (as per strength and frequency) to the top event, a crisp number is calculated for the latter and compared to the specified threshold. 
Likewise, based on a nested loop mechanism, the most influential combination responsible for the top event is experimentally confirmed and, if possible, rectified; otherwise the next combination is examined, and so on until the value of the top event reaches the threshold. The selectivity of research is thereby enhanced considerably by concentrating attention to the most influential variables, consequently saving resources and increasing research value. A measure of the success of the corrective activity is given by the crisp value estimated for the top event, which assumes rather the character of the 'best achievable' than 'analytically correct', thereby providing the background necessary to proceed with wide applicability.

\section{Sensor sensitivity drift}

Sensor sensitivity is a controversial term. It usually implies the slope of the calibration curve in the vicinity of the expected real measurement, i.e., a function of analyte concentration [12]; as such it can be defined as either the minimum input signal required to produced a specific output, given a certain signal-tonoise ratio, or as the ratio of the signal output for a given concentration to the signal obtained for an adjacent concentration value $(\mathrm{C} \pm 1)$. This property is very crucial, especially towards the limit of detection where hypoglycaemic episodes should be identified as soon as possible in order for the patient to receive insulin, as well as for the detection of abrupt glucose changes [2].

A sensitivity drift (set as top event) involves either a responsivity decrease, i.e., a decrease of the output signal in response to input [7, 8], or a modification of the analyte flux kinetics (the pumping of glucose from the interstitial fluid in the sensor) [13], or incorrect calibration, especially in in vivo-ex vivo correlations [9]. The intermediate events that lead to these second-level events can be revealed when considering the design, the operation and the environment around and within the sensor. A sample of the background tree is presented in fig. 1, whereas the description of its nodes is as follows: (1.1) decrease of responsivity; (1.2) modification of analyte flux kinetics; (1.3) poor in vivo/in vitro correlation; (1.1.1) inhibition of enzymatic reaction; (1.1.2) degeneration of enzyme; (1.2.1) decrease of analyte influx to the enzyme; (1.2.2) alteration of the absolute analyte concentration; (1.2.3) alteration of kinetics; (1.3.1) increase of the in vivo basal sensor current; (1.3.2) decrease of the linearity of response; (1.3.3) small frequency of in vitro sensor calibration; (1.1.1.1) oxygen deficit; (1.1.1.2) shortage of supplementary chemicals; (1.1.1.3) electrochemical interference; (1.1.2.1) enzyme poisoning; (1.1.2.2) heat shock; (1.2.1.1) physical barrier to analyte transport; (1.2.1.2) modification of the micro-environmental conditions at the peri-implant site; (1.2.2.1) limitation of the blood supply to the periimplant site; (1.2.2.2) increased extra-sensor analyte consumption; (1.2.2.3) decrease of vascular analyte output; (1.2.2.4) loss of equilibrium between the exudate and blood levels; (1.1.2.1.1) proteolysis induced by exudate proteolytic enzymes entering the reaction chamber; (1.1.2.1.2) from endogenous molecules; 


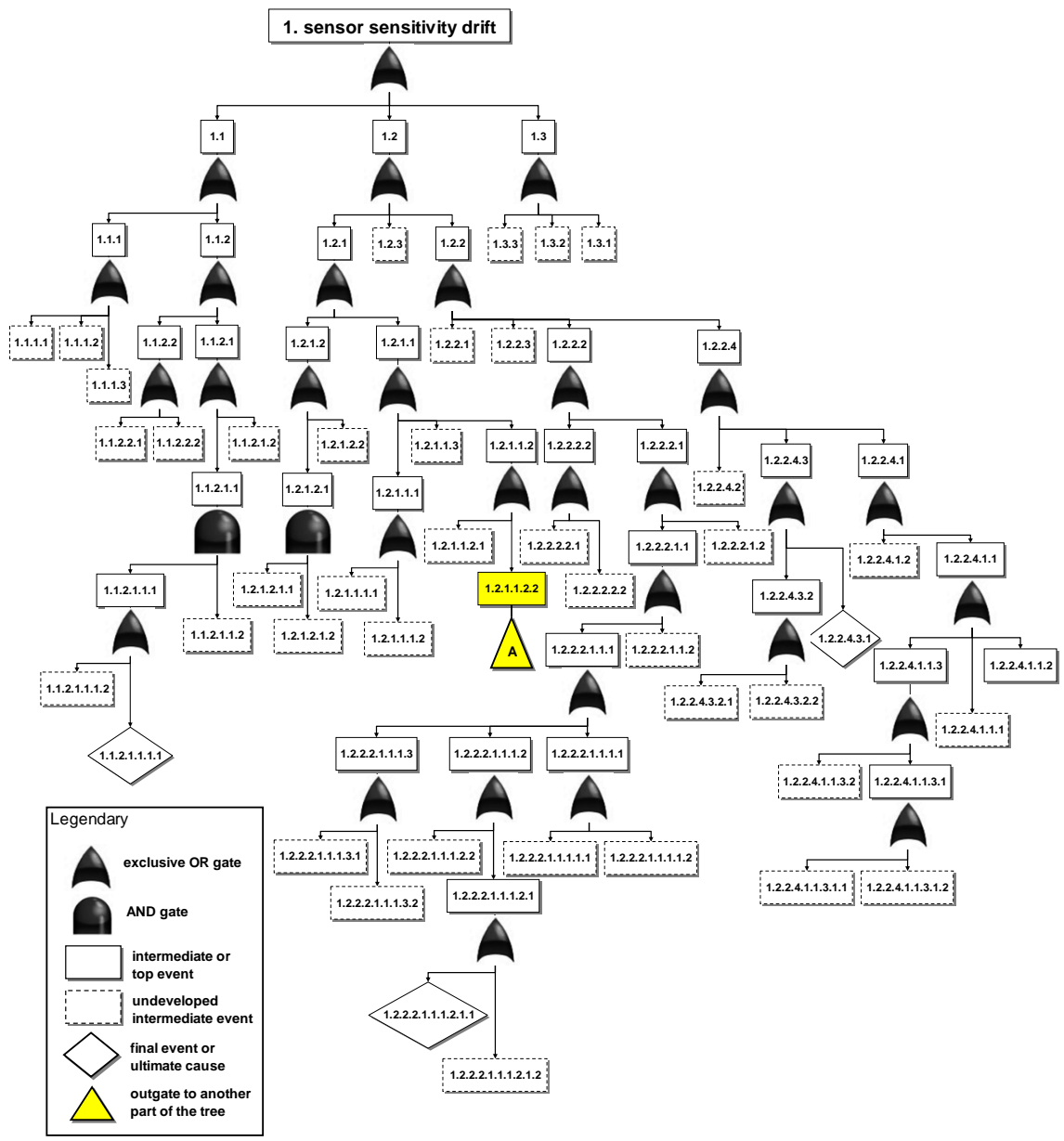

Figure 1: $\quad$ Sample of the fault tree investigating the causes of sensitivity drift in implantable sensors: the qualitative upper part of the tree constituting the content part of the methodological framework (the formal being the mechanism of setting/revising the fuzzy rules).

(1.1.2.2.1) temperature above $37^{\circ} \mathrm{C}$ for a long period of time; (1.1.2.2.2) exothermic reactions induced abrupt temperature increase; (1.2.1.1.1) increase of outer sensor membrane hydrophobicity; (1.2.1.1.2) biofouling of outer sensor membrane; (1.2.1.1.3) tissue encapsulation of the sensor; (1.2.1.2.1) decrease of tissue $\mathrm{pH}$; (1.2.1.2.2) increase of tissue void volume; (1.2.2.2.1) increase of adjacent cells uptake and metabolism; (1.2.2.2.2) endothelial metabolism (wall effect); (1.2.2.4.1) formation of thick fibrous capsule surrounding the implant; (1.2.2.4.2) insufficient vascularisation of the fibrous capsule surrounding the implant; (1.2.2.4.3) rapid changes in blood glucose alteration; (1.1.2.1.1.1) partial disintegration of outer sensor membrane; (1.1.2.1.1.2) increased influx of 
oxygen radicals; (1.2.1.1.1.1) increase of protein exchange between the sensor surface and the exudate; (1.2.1.1.1.2) lipid phase shifts; (1.2.1.1.2.1) excessive protein deposition blocking membrane pores; (1.2.1.1.2.2) thrombus formation on the sensor surface; (1.2.1.2.1.1) increased release of proteolytic enzymes from the exudate cells; (1.2.1.2.1.2) increased release of oxygen radicals from the exudate cells; (1.2.2.2.1.1) excessive numbers of inflammatory cells at the periimplant site; (1.2.2.2.1.2) hormonal activation; (1.2.2.2.2.1) implant touches the blood vessel wall; (1.2.2.2.2.2) vasoconstriction of the artery or vein underlying the implant; (1.2.2.4.1.1) increased fibroblast attachment; (1.2.2.4.1.2) trauma; (1.2.2.4.3.1) glucose infusion; (1.2.2.4.3.2) stress response; (1.1.2.1.1.1.1) membrane stripped during implantation; (1.1.2.1.1.1.2) large fracture leading to local rupture; (1.2.2.2.1.1.1) persisting inflammation; (1.2.2.2.1.1.2) autoimmune response; (1.2.2.4.1.1.1) electric field created around the implant attracts fibroblasts; (1.2.2.4.1.1.2) increase of tissue $\mathrm{pH}$; (1.2.2.4.1.1.3) alteration of oxygen tension at the peri-implant site; (1.2.2.4.3.2.1) increase in the hormonal release; (1.2.2.4.3.2.2) increase of lipid metabolism; (1.2.2.2.1.1.1.1) implant movement; (1.2.2.2.1.1.1.2) toxic shock response to chemicals leaked from the sensor; (1.2.2.2.1.1.1.3) prevention of normal wood healing process; (1.2.2.4.1.1.3.1) increase of oxygen inflow to the sensor; (1.2.2.4.1.1.3.2) increase of the membrane oxygen solubility; (1.2.2.2.1.1.1.1.1) involuntary contraction of the muscle underlying the implant tissue; (1.2.2.2.1.1.1.1.2) collapse of the subcutaneous fibres at the implant site; (1.2.2.2.1.1.1.2.1) partial disintegration of outer sensor membrane; (1.2.2.2.1.1.1.2.2) increase in the release of NO from the vascular endothelial cells; (1.2.2.2.1.1.1.3.1) increase in the release of NO from the vascular endothelial cells; (1.2.2.2.1.1.1.3.2) inhibition of collagen deposition or/and cross-linking; (1.2.2.4.1.1.3.1.1) increase of partial oxygen pressure in the interstitial space; (1.2.2.4.1.1.3.1.2) angiogenesis due to exercise or pharmaceuticals; (1.2.2.2.1.1.1.2.1.1) increased leukotriene synthesis by the activation of the lipossigenesis pathway; (1.2.2.2.1.1.1.2.1.2) alteration of tissue matrix at the peri-implant site.

Each tree node has been quantified using ranges of values that could be described by the linguistic terms (fuzzy sets) low, medium, and high. The degree, or weighting, that any value belongs to a fuzzy set is represented by the membership function; over their domain of interest, the membership functions take a value between 0 and 1 ; each term is schematically presented as a triangle, peaking at the value that the node-event is most commonly found (membership function 1). Using the inference engine, the basal events are entered as crisp numbers (values) and their directly intermediate events (output) are produced as fuzzy sets; these, after defuzzification, they are re-entered as crisp values, and so-forth until a crisp value is produced for the top-event.

\section{Implementation}

The methodology described above has been implemented in node 1.2.1.1.2.2 (branch A of fig. 1), 'thrombus formation on the sensor surface', one of the most commonly encountered problem in implanted materials [5]: the thrombus covers 
the sensor surface inhibiting glucose diffusion (fig. 2). The descriptors to the FTA coding used are: (A.1) platelet adhesion and acceleration; (A.2) increased synthesis of collagen by the fibroblasts, abundant and compact, with the purpose of substituting the damaged tissue; (A.2.1) growth factors produced by the macrophages, exert on the fibroblasts a chemotactic attraction, stimulate their reproduction and induce them to synthesize collagen; (A.2.2) T-lymphocytes produce chemotactic factors and factors capable of increasing the synthesis of collagen by the active fibroblasts; (A.2.1.1) platelet factors attract macrophages and fibroblasts on the peri-implant site; (A.2.1.2) the polymorphonucleated and the macrophage cells, derived from the transformation of the monocytes, exert a phagocytic function serving to remove cellular debris and possibly eliminate the foreign body; (A.2.2.1) the growth factors produced by the macrophages activate T-lymphocytes; (A.2.1.2.1) alteration of tissue matrix at the peri-implant site; (A.2.1.2.2) increased leukotriene synthesis by the activation of the lipossigenesis pathway; (A.2.1.2.1.1) alteration provoked by the liberation of the lysosomal enzymes; (A.2.1.2.1.2) increased presence of leukocytes in the peri-implant site; (A.2.1.2.1.2.1) enlargement of the intercellular junctions permits the leukocytes to pass through; (A.2.1.2.1.2.2) the adhesion of the leukocytes to the endothellial cells is regulated by various types of adhesion molecules which are found on the membranes of both the leukocytes and the endothelial cells; (A.2.1.2.1.2.1.1) histamine, derived from the degranulation of mast cells and chinine, produced by the factor XIIa, provoke vasodilatation and enlargement of the intercellular junctions in the endothelial; (A.2.1.2.1.2.1.2) vasodilatation provokes a slowing of the blood flow that permits leukocytes to migrate towards the margin of the vessel, adhere to the wall and pass through; (A.2.1.2.1.2.2.1) this action is regulated by cytochine which is produced by the fibroblasts situated at the implant site; (A.2.1.2.1.2.1.2.1) increased production of prostacyclin; (A.2.1.2.1.2.1.2.2) lipid infiltration; (A.2.1.2.1.2.1.2.1.1) late response induced by increased production of inteleukin-1; (A.2.1.2.1.2.1.2.1.2) ATP-induced increase in intracellular $\mathrm{Ca}^{2+}$ in venous endothelium promotes prostacyclin synthesis; (A.2.1.2.1.2.1.2.2.1) accumulation of oxidized low-density lipoprotein in the arterial wall; (A.2.1.2.1.2.1.2.2.2) vitamin A deficiency; (A.2.1.2.1.2.1.2.2.3) ageing of the vascular tissue; (A.2.1.2.1.2.1.2.1.1.1) high daily doses of vitamin $C$ intake; (A.2.1.2.1.2.1.2.1.1.2) histiocytic chronic phlogosis; (A.2.1.2.1.2.1.2.1.2.1) activation of $\mathrm{Ca}^{2+}$ release from $\mathrm{Ca}^{2+}$ stores; (A.2.1.2.1.2.1.2.1.2.2) $\mathrm{Ca}^{2+}$ influx through ion channels in the plasma membrane; (A.2.1.2.1.2.1.2.2.1.1) high plasma cholesterol level; (A.2.1.2.1.2.1.2.2.1.2) long duration of hypercholesteremic period; (A.2.1.2.1.2.1.2.1.2.2.1) increased calcium intake; (A.2.1.2.1.2.1.2.1.2.2.2) hypecalcaemia; (A.2.1.2.1.2.1.2.1.2.2.1.1) as a treatment course for osteoporosis; (A.2.1.2.1.2.1.2.1.2.2.1.2) as supplement intake; (A.2.1.2.1.2.1.2.1.2.2.2.1) due to recurrent adenocarcinoma; (A.2.1.2.1.2.1.2.1.2.2.2.2) due to constitutive activity of the parathyroid hormone (PTH)/PTH-related peptide receptor.

The reactions of the body to an implant crucially depend on the surface of the implant [4], especially the chemical situation present at the surface [5], the surface texture [4], the local flow conditions [13], as well as other factors. It is 
104 Modelling in Medicine and Biology X

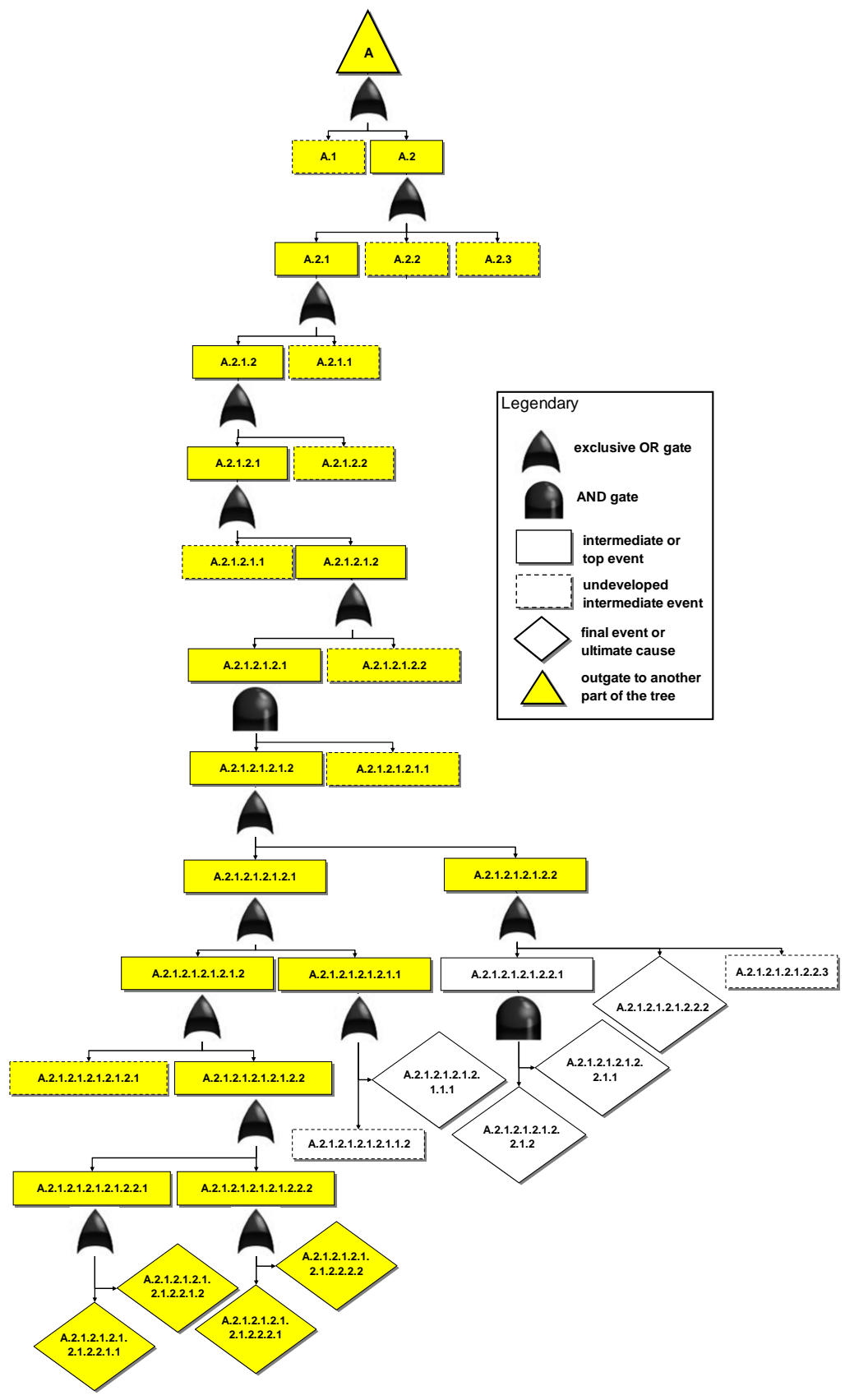

Figure 2: $\quad$ Part of the 1.2.1.1.2.2 (A-branch), referring to thrombus formation on the sensor surface. 
Table 1: $\quad$ The degree and extent of contribution of some of the parameters that directly or indirectly lead to thrombus formation on the sensor surface (node A), estimated through the inference achieved by the chain of fuzzy rules, leading from the basal event of node A.2.1.2.1.2.1.2.1.2.2.1.1 to event A.

\begin{tabular}{|l|c|c|c|c|c|c|c|c|}
\hline NODE No & $\# 1$ & $\# 2$ & $\# 3$ & $\# 4$ & $\# 5$ & $\# 6$ & $\# 7$ & $\# 8$ \\
\hline A.2.1.2.1.2.1.2.1.2.2.1.1 & 180 & 550 & 1200 & 180 & 1200 & 180 & 180 & 2500 \\
\hline A.2.1.2.1.2.1.2.1.2.2.2 & 5 & 5 & 5 & 5 & 5 & 5 & 5 & 5 \\
\hline A.2.1.2.1.2.1.2.1.2.1 & 8 & 8 & 8 & 50 & 8 & 8 & 8 & 8 \\
\hline A.2.1.2.1.2.1.2.1.1 & 4 & 4 & 4 & 4 & 4 & 4 & 4 & 4 \\
\hline A.2.1.2.1.2.1.2.2 & 6 & 6 & 6 & 6 & 70 & 10 & 10 & 10 \\
\hline A.2.1.2.1.2.1.1 & 1 & 1 & 1 & 1 & 1 & 18 & 160 & 18 \\
\hline A.2.1.2.1.2.2 & 4 & 4 & 4 & 4 & 4 & 4 & 4 & 4 \\
\hline A.2.1.2.1.1 & 2 & 2 & 2 & 2 & 2 & 2 & 2 & 2 \\
\hline A.2.1.2.2 & 6 & 6 & 6 & 6 & 6 & 6 & 6 & 6 \\
\hline A.2.1.1 & 4 & 4 & 4 & 4 & 4 & 4 & 4 & 4 \\
\hline A.2.2 & 12 & 12 & 12 & 12 & 12 & 12 & 12 & 12 \\
\hline A.2.3 & 14 & 14 & 14 & 14 & 14 & 14 & 14 & 14 \\
\hline A.1 & 8 & 8 & 8 & 8 & 8 & 8 & 8 & 8 \\
\hline Output of A (\%) & 7.33 & 7.52 & 7.65 & 7.98 & 7.85 & 22.67 & 22.89 & 22.91 \\
\hline
\end{tabular}

generally known, that 'increased platelet adhesion, activation and aggregation' (node A.1) on implant surfaces exposed precede the formation of the thrombus [14]. Despite, however, the directly related physiological reasons that contribute to the top even, implant failure can be triggered by phenomenologically unrelated reasons, as calcium or vitamin $C$ intake $[15,16]$ (nodes A.2.1.2.1.2.1.2.1.2.2.1 and A.2.1.2.1.2.1.2.1.1.1, respectively), vitamin A deficiency (node A.2.1.2.1.2.1.2.2.2) or hypercholesteraemia. The former could be of significant importance, since most type II patients suffer from severe osteoporosis, requiring chronic treatment.

A sample of the fuzzy rules used, representing a chain leading from the eighteen-digit final event A.2.1.2.1.2.1.2.1.2.2.1.1 to A (i.e., the six-digit intermediate event 1.2.1.1.2.2), indicated by the highlighted path in fig. 2, is presented below:

IF A.2.1.2.1.2.1.2.1.2.2.1 is [low] AND A.2.1.2.1.2.1.2.1.2.2.2 is [medium] THEN A.2.1.2.1.2.1.2.1.2.2 is [medium].

IF A.2.1.2.1.2.1.2.1.2.1 is [high] AND A.2.1.2.1.2.1.2.1.2.2 is [medium] THEN A.2.1.2.1.2.1.2.1.2 is [high].

IF A.2.1.2.1.2.1.2.1.1 is [low] AND A.2.1.2.1.2.1.2.1.2 is [high] THEN A.2.1.2.1.2.1.2. is [medium]. 

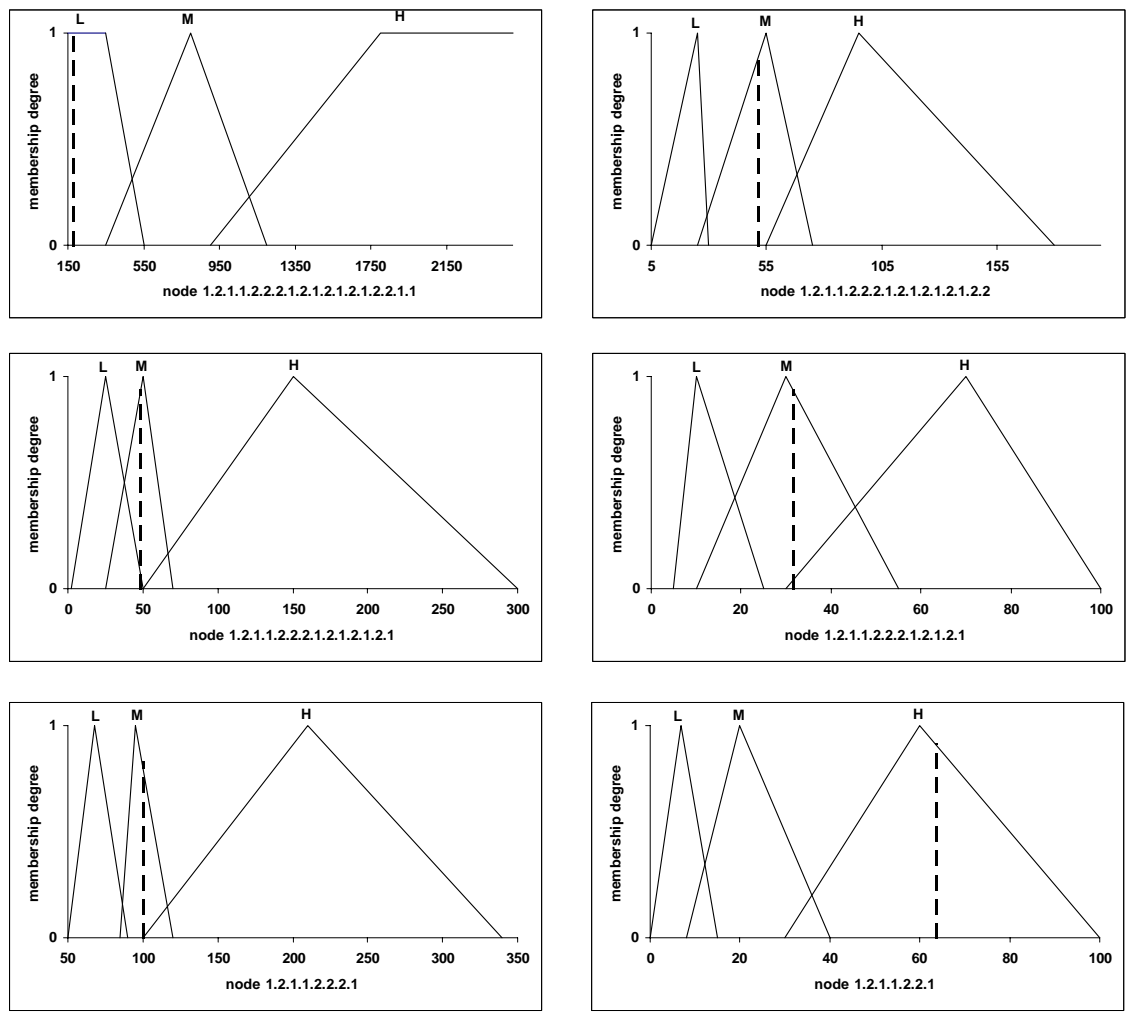

Figure 3: Membership function of some of the variables participating within the fuzzy chain leading from the final event A.2.1.2.1.2.1.2.1.2.2.1.1 (increased calcium intake by the subject as a treatment course for osteoporosis) to event A (or 1.2.1.1.2.2: thrombus formation on the sensor surface), when all other input variables are in the medium range. The dashed line represents output values (as shown in case 9 of Table 1), except from the basal event (input value).

The threshold value set for event A is $20 \%$, as proven suitable for retaining much of sensor's functionality. The problem investigated refers mainly to the effect of calcium intake by the subject upon the sensor performance, considering also other parallel physiological processes. Nine scenarios have been tested (Table 1) aiming at examining all possible combinations and influences. The results have shown that at any value of the basal event (cases 1-3), event A is well below the tolerance value, provided that all other inputs are kept low. There is a slight increase in event $A$ when the calcium stores are activated (node A.2.1.2.1.2.1.2.1.2) (case 4), as well as at increased lipid infiltration (node A.2.1.2.1.2.1.2.2) (case 5), mainly due to high cholesterol levels (node A.2.1.2.1.2.1.2.2.1.1). At cases 6-8, even when histamine levels are kept at 
medium values, the estimated values are above the set threshold. It can be thus concluded that, after implantation, the cholesterol and histamine levels should be carefully monitored at a regular basis in order to prevent thrombogenic activity.

\section{Discussion and conclusions}

The method presented herein serves effectively to localize the causes of sensor drift in implantable biosensors under conditions of uncertainty. When coupled to fuzzy reasoning, it provides a tool for internal diagnostics and fault compensation under nearly real-time conditions. The FTA approach suggested enhances selectivity of $R \& D$ effort by concentrating attention to the most influential variables, consequently saving valuable resources (time, effort, money, scientific equipment, highly skilled labour). When investigating biosensor failure during in vivo operation, feasibility is enhanced by assigning threshold values of the tolerability of the device to the top event. In this way, the required distinction between critical and non-critical status of the top event can be sharper to improve sensor validation, an issue of great significance, especially for biomedical applications intended for everyday, wide-spread self-monitoring uses. The approach suggested may contribute significantly to the selfoptimisation of the measuring equipment from one generation to the next as it supports the flexible ad hoc tailor made development, thus potentiating the progress of epidemics from statistics to individualisation.

\section{Acknowledgement}

Financial support provided by the Research Centre of the University of Piraeus is kindly acknowledged.

\section{References}

[1] Klueh, U., Dorsky, D.I. and Kreutzer, D.L., Enhancement of implantable glucose sensor function in vivo using gene transfer-induced neovascularisation. Biomaterials, 26, pp. 1155-1163, 2005.

[2] Wickramasinghe, Y., Yang, Y. and Spencer, S.A., Current problems and potential techniques in in vivo glucose monitoring. Journal of Fluorescense, 14, pp. 513-520, 2004.

[3] Nablo, B.J., Prichard, H.L., Butler R.D., Klitzman B. and Schoenfisch, M.H., Inhibition of implant-associated infections via nitric oxide release. Biomaterials, 26, pp. 6984-6990, 2005.

[4] Berglin, M., Andersson, M., Sellborn, A. and Elwing, H., The effect of substrate molecular mobility on surface induced immune complement activation and blood plasma coagulation. Biomaterials, 25, pp. 4581-4590, 2004.

[5] Faucheux, N., Tzovena, R., Nagel, M.-D. and Groth T., The dependence of fibrillar adhesions in human fibroblasts on substratum chemistry, Biomaterials, 27, pp. 234-245, 2006. 
[6] Wisniewski, N. and Reichert, M., Methods for reducing biosensor membrane biofouling. Colloids and Surfaces B: Biointerfaces, 18, pp. 197219, 2000.

[7] Batzias, F.A. and Siontorou, C.G., Investigating the causes of biosensor SNR decrease by means of fault tree analysis. IEEE Transactions on Instrumentation and Measurement, 54, pp. 1395-1406, 2005.

[8] Siontorou, C.G., Batzias, F.A. and Tsakiri, V., A knowledge-based approach to online fault diagnosis of FET biosensors. IEEE Transactions On Instrumentation And Measurement, 59, pp. 2345-2364, 2010.

[9] Jeong, R.-A., Hwang, J.Y., Joo, S., Chung, T.D., Park, S., Kang, S.K., Lee, W.-Y. and Kim, H.C., In vivo calibration of the subcutaneous amperometric glucose sensors using a non-enzyme electrode. Biosensors and Bioelectronics, 19, pp. 131-139, 2003.

[10] Banerjee, R., Nag, S. and Fraser, H.L., A novel combinatorial approach to the development of beta titanium alloys for orthopaedic implants. Materials Science and Engineering C, 25, pp. 282-289, 2005.

[11] Bellazzi, R., Guglielmann, R. and Ironi, L., Learning from biomedical time series through the integration of qualitative models and fuzzy systems. Artificial Intelligence in Medicine, 21, pp. 215-220, 2001.

[12] Thévenot, D.R., Toth, K., Durst, R.A. and Wilson, G.S., Electrochemical biosensors: recommended definition and classification. Pure and Applied Chemistry, 71, pp. 2333-2348, 1999.

[13] Usher, M.J., Sensors and Transducers, Macmillan: London, UK, 1985.

[14] Theoret, C.L., Update on wound repair. Clinical Techniques in Equine Practice, 3, pp. 110-122, 2004.

[15] Choi, J., Hammer, L.W. and Hester, R.L., Calcium-dependent synthesis of prostacyclin in ATP-stimulated venous endothelial cells. Hypertension, 39, pp. 581-585, 2002.

[16] Deng, T., Yu, L., Ge, Y., Zhang, L. and Zheng, X., Intracellular-free calcium dynamics and F-actin alteration in the formation of macrophage foam cells. Biochemical and Biophysical Research Communications, 338, pp. 748-756, 2005. 